\title{
NOUVELle
}

\section{Un facteur de transcription se fait complice du VIH-1 pour détruire les défenses cellulaires}

Roland Marquet, Santiago Guerrero, Serena Bernacchi, Sophie Pernot, Julien Batisse, Jean-Christophe Paillart

> Les éléments génétiques mobiles représentent une fraction considérable de notre génome et les mammifères ont développé plusieurs facteurs cellulaires restreignant l'expansion des transposons, rétrotransposons et rétrovirus. Plusieurs facteurs de restriction inhibant la réplication des rétrovirus, dont les virus de l'immunodéficience humaine de types 1 et 2 ( $\mathrm{VIH}-1, \mathrm{VIH}-2)$, ont ainsi été identifiés: TRIM5 $\alpha$ (tripartite motif-containing protein $5 \alpha$ ), la téthérine, SAMHDI (SAM domain and HD domain 1), et plusieurs membres de la famille des cytidines désaminases APOBEC-3 (apolipoprotein B mRNAediting, enzyme-catalytic, polypeptide-like 3) [1-3]. Parmi ces derniers, $A-3 G$ et $A-3 F$, qui sont exprimés dans les cellules cibles naturelles des VIH-I et $\mathrm{VIH}-2$, sont les plus importants $[1,3]$. Les rétrovirus ont cependant acquis la capacité de contourner ces facteurs de restriction. Certains ont développé des protéines dites accessoires dans ce seul but : c'est le cas du facteur d'infectiosité viral (Vif) et de la protéine virale U (Vpu) des VIH-l et $\mathrm{VIH}-2$, ainsi que de la protéine virale $X(V p x) d u$ VIH-2, qui contrecarrent respectivement $A-3 C / 3 D E / 3 F / 3 G / 3 H$ $[1,3]$, la téthérine [1] et SAMHDI [2]. La neutralisation des facteurs de restriction par les rétrovirus implique très souvent le détournement de la voie de dégradation des protéines cellulaires par le protéasome en réponse à leur polyubiquitination.
Ubiquitinylation des facteurs de restriction par la protéine virale Vif L'ubiquitinylation des protéines eucaryotes fait tout d'abord intervenir une des deux enzymes activatrices, $\varepsilon l$, qui transfère l'ubiquitine à l'une des enzymes de conjugaison, દ2 ; enfin, une ubiquitine ligase ( $\varepsilon 3$, il en existe plusieurs centaines), transfère l'ubiquitine aux substrats spécifiques. Trois protéines accessoires du VIH-1, Vif, Vpu et $\mathrm{Vpr}$ sont connues pour détourner trois ubiquitine ligases $\varepsilon 3$ différentes appartenant à la famille des cullin-RING $\varepsilon 3$ ligases. La protéine Vif du VIH-l possède en son centre un motif $\mathrm{HCCH}$ liant le zinc et une boîte culline qui permettent le recrutement de la culline 5 ( Cul-5), Cul-5 étant capable de lier Rbx-2, une protéine possédant un domaine RING. Vif possède en outre dans sa région carboxy-terminale un motif appelé SOCS (suppressor of cytokine signaling) box qui recrute l'élongine $C(\varepsilon l o-C)$ qui forme un hétérodimère avec l'élongine $\mathrm{B}$ (Elo-B) (Figure 1, voie B). Le complexe $\mathrm{Cul}-5 / \mathrm{Rb} \times 2 / \varepsilon \mid \mathrm{l}-\mathrm{B} / \varepsilon \mathrm{ll}_{0}-\mathrm{C}$ ainsi recruté par la protéine Vif permet l'ubiquitinylation d'A-3G/A-3F, orientant ainsi ces protéines vers la dégradation par le protéasome (Figure 1, voie B).

\section{CBF- $\boldsymbol{\beta}$ : un nouveau ligand de Vif} impliqué dans l'infectiosité des VIH/VIS chez les primates Deux articles publiés récemment dans la revue Nature $[4,5]$ ont identifié un nouveau ligand de Vif impliqué dans la
Architecture et réactivité de l'ARN, Université de Strasbourg, CNRS, Institut de biologie moléculaire et cellulaire, 15 , rue René Descartes, 67084 Strasbourg cedex, France. r.marquet@ibmc-cnrs.unistra.fr

dégradation d'A-3G, qui fait également l'objet d'une publication récente dans Journal of Virology [6]. En effet, Krogan et al. [7] ont entrepris l'identification systématique des ligands des protéines du VIH-1, dont Vif, en combinant purification d'affinité et spectrométrie de masse. Chacune des protéines du VIH-l a été exprimée avec deux étiquettes différentes (Strep ou Flag) soit par transfection transitoire de cellules humaines d'épithélium rénal embryonnaire (HEK-293), soit par expression stable et inductible à partir de cellules lymphoïdes Jurkat. Les auteurs se sont focalisés sur les protéines identifiées indépendamment du type cellulaire et de l'étiquette utilisée pour la purification $[5,7]$. Par cette approche, sept protéines humaines ont été identifiées comme des ligands directs ou indirects de Vif dans les deux types cellulaires: I'une intervient dans l'autophagie (MARAl), cinq sont les constituants de l'ubiquitine ligase \&3 (દlo-B, Elo-C, Cul5 , Rbx-2, et Cul-2 qui interagit avec Cul5 ) et la dernière est CBF- $\beta$ (core binding factor- $\beta$ ). Zhang et al. [4] ont, quant à eux, employé Vif munie d'une étiquette HA (hémaglutinine) pour immunoprécipiter ses partenaires dans les cellules $\mathrm{H} 9$, une lignée cellulaire T. Ils ont ensuite identifié par spectrométrie de masse Cul-5, Elo-B, Elo-C et CBF- $\beta$. L'interaction entre Vif et CBF- $\beta$ a été validée par des études de colocalisation et de transfert d'énergie de fluorescence (FRET) dans des cellules HEK 293T transfectées [4]. 


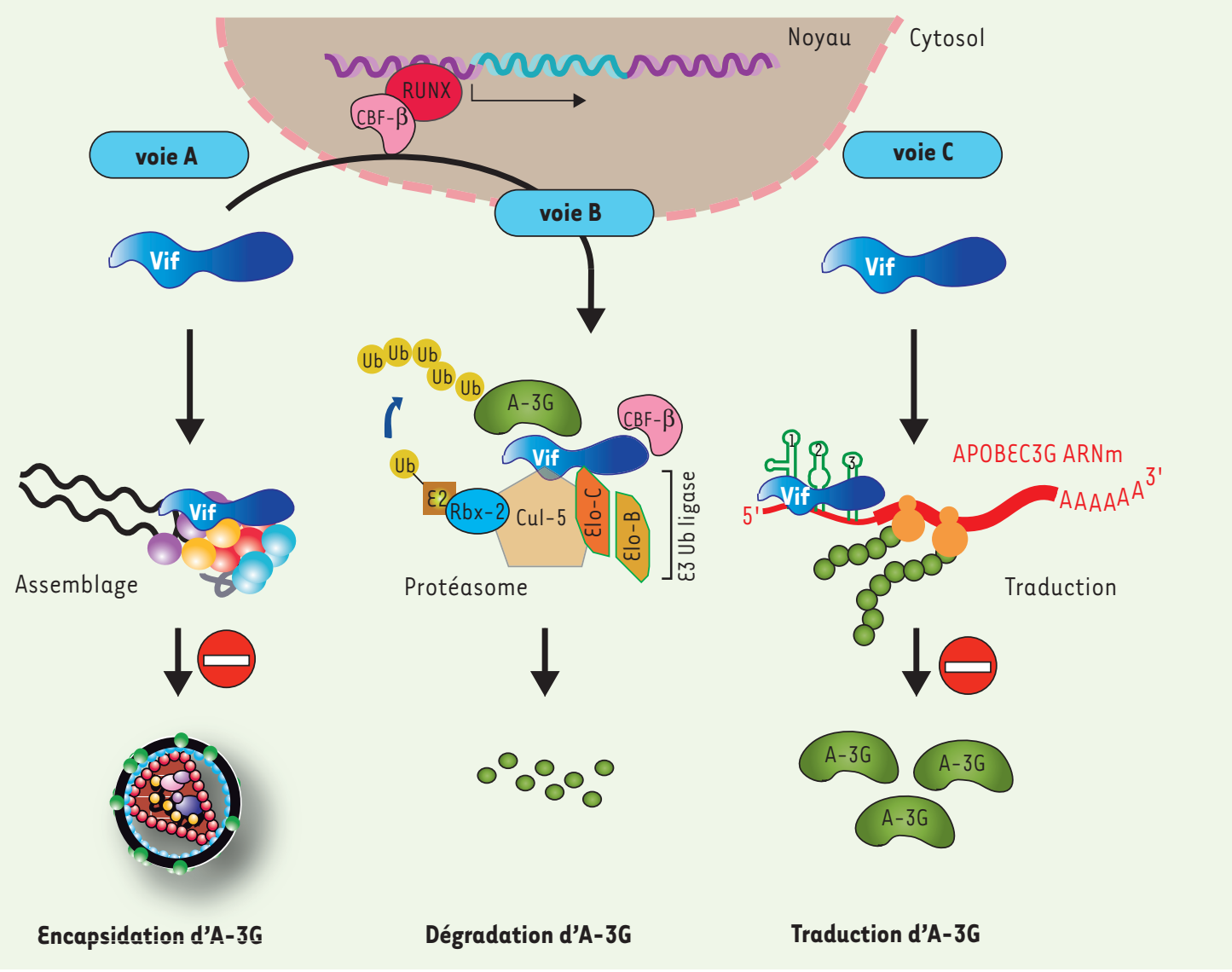

Figure 1. Mécanismes d'action de Vif. Vif contrecarre le facteur de restriction A3-G par au moins trois mécanismes distincts : (1) compétition avec A-3G lors de l'assemblage viral (voie A), (2) induction de sa dégradation par le protéasome (voie B), et (3) inhibition de sa traduction (voie C).

CBF- $\beta$ est un cofacteur de transcription impliqué dans le développement et la différenciation de plusieurs types cellulaires, dont les lymphocytes T. II ne lie pas I'ADN mais interagit avec les protéines de la famille RUNX (Runt-related transcription factor 1 ), dont il module le repliement et les propriétés de fixation à l'ADN (Figure 1). Zhang et al. ont déterminé que la région amino-terminale de Vif est cruciale pour sa liaison à $C B F-\beta$ et que cette liaison n'entre pas en compétition avec le recrutement des protéines RUNX dont Vif n'affecte pas l'activité transcriptionnelle [4]. Les deux publications parues dans Nature $[4,5]$ démontrent que CBF- $\beta$ fait partie du complexe $\varepsilon 3$ ubiquitine ligase comprenant la Cul-5, Rbx2, Elo-C et $\varepsilon l o-B$. Cependant, la fixation de CBF- $\beta$ à ce complexe requiert $V$ if, ce qui indique que CBF- $\beta$ n'est pas un constituant général des Cul-5 83 ubiquitine ligases $[4,5]$. Vif forme un complexe soluble et monodisperse avec CBF- $\beta$, Elo-B et Elo-C qui lie $A-3 G$ et s'associe à Cul-5/ Rxb-2 [5], CBF- $\beta$ facilitant le recrutement de Cul-5 [4]. La réciproque n'est cependant pas vraie: Cul-5 n'influence pas la fixation de CBF- $\beta$ à Vif [4]. En outre, Jäger et al. ont démontré que le complexe Cul-5/Rxb-2/Elo-B/Elo-C/Vif/ $\mathrm{CBF}-\beta$ reconstitué in vitro à partir de protéines recombinantes est capable de polyubiquitiner A-3G [5].

Les deux études $[4,5]$ ont démontré également que lorsque l'expression de CBF- $\beta$ est diminuée par l'utilisation de SIARN ou de shARN, la concentration intracellulaire d'A-3G et son incorporation dans les particules virales nécessaire à son activité de restriction - augmentent, tandis que l'infectiosité du VIH-l diminue. Comme attendu,
CBF- $\beta$ n'affecte pas l'infectiosité virale en l'absence d'A-3G. L'étude de Hulquist et al. étend la portée de ces résultats, puisqu'elle les généralise aux deux isoformes de CBF- $\beta$, à un grand nombre d'isolats du VIH-l de sous-types différents et à l'ensemble des cytidines désaminases de la famille APOBEC 3 sensibles à la dégradation induite par Vif $(A-3 C / 3 D E / 3 F / 3 G / 3 H)$ [6]. De façon similaire, $C B F-\beta$ lie la protéine Vif du virus d'immunodéficience des macaques rhésus (VISmac), favorisant la dégradation des APOBEC -3 sensibles à Vif chez cet animal et leur exclusion des particules virales dont elle augmente l'infectiosité [4-6]. Cependant, la dégradation de l'unique protéine APOBEC-3 bovine induite par Vif du virus de l'immunodéficience bovine (VIB) n'est pas affectée par CBF- $\beta$ [4], suggérant que 
l'implication de cette protéine dans la dégradation des protéines de la famille APOBEC -3 se limite aux primates.

\section{Perspectives thérapeutiques antivirales}

L'identification d'un facteur humain intervenant spécifiquement dans la dégradation des protéines de la famille APOBEC-3 ouvre la voie à de nouvelles stratégies antivirales dirigées contre les VIH-1 et 2. En effet, des molécules perturbant l'interaction entre Vif et CBF- $\beta$ devraient inhiber la dégradation de ces facteurs de résistance sans perturber la fonction des autres ubiquitine ligases. La mise en évidence $\mathrm{du}$ complexe stable et soluble CBF- $\beta$ /Vif/Elo-C/Elo-B et la reconstitution in vitro d'un complexe $\varepsilon 3$ ubiquitine ligase actif [5] permettent d'envisager le criblage à haut flux d'inhibiteurs ou leur conception rationnelle lorsque la structure cristallographique de ces complexes aura été obtenue. La dégradation d'A-3G par la voie du protéasome n'est pas le seul mécanisme par lequel Vif contrecarre ce facteur de restriction : Vif réduit également l'expression d'A-3G en inhibant sa traduction [8] (Figure 1, voie C) et agit de façon directe pour inhiber son incorporation dans les particules virales, sans doute par compétition [3] (Figure 1, voie A). Ces deux derniers mécanismes restent encore mal connus et pourraient, eux-aussi, déboucher sur de nouvelles stratégies d'inhibition du VIH-1. 0

\section{A transcription factor acts as} a HIV-1 accomplice to destroy the cellular defences

\section{RÉFÉRENCES}

1. Strebel K, Luban J, Jeang KT. Human cellular restriction factors that target HIV-1 replication. BMC Med $2009 ; 7: 48$.

2. Laguette N, Sobhian B, Casartelli N, et al. SAMHDl is the dendritic- and myeloid-cell-specific HIV-1 restriction factor counteracted by Vpx. Nature 2011 ; 474 : 654-7.

3. Henriet S, Mercenne G, Bernacchi S, et al. Tumultuous relationship between the human immunodeficiency virus type 1 viral infectivity factor (Vif) and the human APOBEC-3G and APOBEC-3F restriction factors. Microbiol Mol Biol Rev $2009 ; 73$ : 211-32.

4. Zhang W, Du J, Evans SL, et al. T-cell differentiation factor CBF-beta regulates HIV-1 Vif-mediated evasion of host restriction. Nature 2012 ; 481 : 376-9.

5. Jager S, Kim DY, Hultquist JF, et al. Vif hijacks CBFbeta to degrade APOBEC3G and promote HIV-1 infection. Nature $2012 ; 481$ : 371-5.

6. Hultquist JF, Binka M, LaRue RS, et al. Vif proteins of human and simian immunodeficiency viruses require cellular CBF- $\beta$ to degrade $A P O B E C 3$ restriction factors. J Virol $2012 ; 86: 2874-7$.

7. Jager $S$, Cimermancic P, Gulbahce N, et al. Global landscape of HIV-human protein complexes. Nature $2012 ; 481: 365-70$.

8. Mercenne G, Bernacchi S, Richer D, et al. HIV-1 Vif binds to APOBEC3G mRNA and inhibits its translation. Nucleic Acids Res 2010 ; 38 : 633-46.

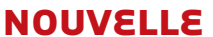

\section{Rôle des fibrilles amyloïdes dans la transmission du VIH}

Nadia R. Roan, Marielle Cavrois, Warner C. Greene
Gladstone Institute of Virology and Immunology, University of California at San Francisco, San Francisco, CA 94158, États-Unis. nroan@gladstone.ucsf.edu
> Comme en témoignent les 34 millions de personnes infectées en 2010, le VIH/ Sida demeure un problème de santé publique majeur [1]. Une grande partie des infections mondiales se concentrent en Afrique sub-saharienne où la transmission lors de rapports hétérosexuels est le mode de propagation le plus courant. Comme cette transmission se produit quasiment toujours en présence de sperme, il est essentiel d'élucider l'effet du sperme sur la transmission du virus.

\section{Le sperme, un facteur facilitateur} de l'infection VIH

Loin d'être un véhicule passif pour le VIH, le sperme peut grandement augmenter l'infection par le VIH in vitro. Plusieurs groupes ont publié que la présence de sperme augmente dramatiquement l'infection de lignées cellulaires et de cellules primaires par le VIH [2-6]. Quels sont les facteurs présents dans le sperme responsables de cet effet ? Un certain nombre de composants du liquide séminal, dont la fonction physiologique est de protéger les spermatozoïdes lors de leur voyage vers l'ovule, peuvent protéger également les virions. C'est le cas des amines basiques comme la spermine, la spermidine, putrescine, cadavérine, etc., qui évitent la dénaturation des spermatozoïdes dans l'environnement acide de l'appareil vaginal et qui, malheureusement, empêchent aussi l'inactivation du virus [7-9]. D'autres composants du sperme, plus récemment identifiés par Munch et al. forment des fibrilles amyloïdes capables d'augmenter l'infectivité du VIH [2]. Ces fibrilles ont été nommées
SEVI pour semen-derived enhancer of viral infection. L'augmentation du pouvoir infectieux du VIH conférée par SEVI est plus marquée lorsque l'inoculum viral est limité, une situation qui semble survenir lors de la transmission du VIH. Des expériences de dilution limite du virus ont montré que les fibrilles de SEVI peuvent augmenter l'infection par un facteur de 100000 [2]. Une dose de VIH qui serait donc insuffisante pour être infectieuse pourrait devenir, dans ce contexte, hautement infectieuse.

\section{Les fibrilles amyloïdes du sperme}

Les fibrilles amyloïdes sont des polymères de feuillets $\beta$ et ont été déjà associées à des maladies neurologiques, maladies d'Alzheimer et de Huntington par exemple. SEVI est constitué de 